www.jmscr.igmpublication.org

Impact Factor 5.84

Index Copernicus Value: 83.27

ISSN (e)-2347-176x ISSN (p) 2455-0450

crossref DOI: _https://dx.doi.org/10.18535/jmscr/v5i6.188

Journal Of Medical Science And Clinical Research

IGM Publication

An official Publication of IGM Publication

\title{
Outcome of Idiopathic Pulmonary Fibrosis and Non-IPF interstitial Lung Diseases at 6 Months in A Tertiary Care Center A Comparative Study
}

\author{
Authors \\ Soofiya.M1 ${ }^{1}$,Sreekala $C^{1}$, Sanjeev Nair ${ }^{1}$, K AnithaKumari ${ }^{1}$ \\ ${ }^{1}$ Department of Pulmonary Medicine, Medical College, Trivandrum, \\ Corresponding Author \\ Dr Sreekala C \\ Navaneetham, APRA 307, Ajantha Pulli Lane, Pettah PO, Chackai, Trivandrum 695024 \\ Email:drkalamurali@yahoo.co.in
}

\begin{abstract}
Introduction: Interstitial lung diseases are a group of diffuse parenchymal lung disorders associated with substantial morbidity and mortality and pose diagnostic and therapeutic challenges to the clinician. Diagnosis of ILD is by multidisciplinary discussion (MDD) between clinician, radiologist and pathologist. Risk prediction is challenging in ILDs because of heterogeneity in disease-specific and patient-specific variables. Studies assessing factors associated with mortality in ILDs are scarce in our setting. Prospective disease registries can provide better estimates of incidence and prevalence as well as insights to etiology, associated risks, natural history, and outcomes of a disease. Data regarding the use of composite prediction models like Composite physiologic index and ILD Gap index are limited.

Aim of study 1.To compare the adverse outcome, as defined by>10\% decline in FVC and mortality at 6 months in patients with Idiopathic pulmonary fibrosis (IPF) and other non IPF interstitial lung diseases diagnosed by $M D D$, at Department of Pulmonary medicine, $M C H$, Trivandrum over a period of two years. Secondary objectives were to determine factors affecting adverse outcomes in interstitial lung diseases and to evaluate the prognostic value of composite physiologic index(CPI) and ILD - GAP index in interstitial lung diseases.

Study design: Prospective Cohort study.

Study setting: Department of Pulmonary Medicine, Government Medical College Trivandrum. Study period: Two years.

Study population: Patients with multidisciplinary diagnosis of ILD, attending Department of Pulmonary Medicine, Government Medical College Trivandrum, Inclusion criteria: All patients diagnosed as ILD patients, who give consent to participate in the study. Exclusion criteria: ILD Patients with Lung Cancer or Pulmonary Tuberculosis. Data collection A prospective cohort study over a period of two years conducted at Pulmonary medicine, Department, Medical College, Thiruvananthapuram. 164 consecutive patients with multidisciplinary diagnosis of interstitial lung disease were included in the study. Detailed history, spirometry, SPO2, DLCO, HRCT, 6 minute walk test, ECG,2D ECHO were done and recorded in duly filled proforma. ANA profile, serum angiotensin converting enzyme, serum calcium, 24 hour urinary calcium were estimated wherever it was indicated. Diagnosis of IPF was done after excluding the known causes and the HRCT findings suggestive of UIP pattern based on the current guidelines of the American Thoracic Society/European
\end{abstract}




\section{JMSCR Vol||05||Issue ||06||Page 23922-23929||June}

Respiratory Society CPI was calculated from spirometry and DLCO. ILD GAP index was also calculated. Patients were given appropriate therapy as per the clinical subtypes. Patients were followed up with clinical assessment and spirometry, and DLCO at 6 months. Outcome variables assessed were death and FVC decline $\geq 10 \%$ at 6 months.

Institutional ethical committee clearance was obtained before data collection. Statitical Analysis. Data were entered in Microsoft Excel and analyzed using Epi Info version 7. For descriptive statistics, quantitative variables were described by mean and standard deviation. Qualitative variables were described by percentage distribution. For inferential statistics between groups, comparison of qualitative variables were analysed by chi-square test and quantitative variables were compared by student $t$ test. $P$ value of less than 0.05 was considered as level of significance.

Survival pattern was assessed by Kaplan-meir survival plot.

Results: Out of the 164 patients studied, 26.2\% were idiopathic pulmonary fibrosis (IPF) and $73.78 \%$ were non-IPF.15.2\% died at 6 months follow up, of which 72\% were IPF. 51.2\% had a fall in FVC $\geq 10 \%$ at 6 months. In bivariate analysis, factors associated with mortality in interstitial lung diseases are: age > 60, male gender, BMI $<18.5$, smoking, presence of pulmonary hypertension, mean saturation $<90 \%$, \% predicted FVC $<50 \%$, \% predicted DLCO $<40 \%$, 6 Minute walk distance $<250$ m, at diagnosis, UIP pattern in HRCT, CPI score > 50 and ILD-GAP index > 4. On multivariate analysis using logistic regression, factors associated with mortality were age > 60 years and IPF group. Similar Factors were associated with decline in FVC more than 10\% at 6 months in bivariate analysis, whereas On multivariate analysis are history of smoking and initial \% predicted DLCO $<40 \%$ were significant. ILD Gap index is a better predictor of mortality than Composite physiologic index in interstitial lung diseases. (AUROC 0.912 vs 0.856 for CPI).

Conclusion: The factors associated with mortality in ILD were age $>60$ years, type of ILD as idiopathic pulmonary fibrosis and decline in FVC $\geq 10 \%$ at 6 month. and FVC $<50 \%$ at baseline. The availability of reliable prediction models like composite physiologic index (CPI) and ILD Gap index can help in prognostication and making clinical decisions. ILD Gap index was a better predictor of mortality than composite physiologic index.

Keywords: Interstitial lung diseases, adverse outcomes, mortality, FVC decline, composite physiologic index, ILD Gap index.

\section{Introduction}

Interstitial lung diseases are a group of diffuse parenchymal lung disorders associated with substantial morbidity and mortality and pose diagnostic and therapeutic challenges to the clinician. Diagnosis of ILD is by multidisciplinary discussion (MDD) between clinician, radiologist and pathologist ${ }^{[1]}$. Risk prediction is challenging in ILDs because of heterogeneity in diseasespecific and patient-specific variables.

FVC may be the most appropriate single prognostic parameter, given its ease of measurement, reproducibility, and ability to predict prognosis at baseline and over time, with even minor changes providing prognostic information ${ }^{[2]}$ FVC was the measure most favored as an outcome for both CTD-ILD and IPF, with $100 \%$ acceptance among the consensus panelists. Changes in FVC, as a potential surrogate for mortality or conversely progression free survival is being explored but currently supported by inconsistent data $^{[3]}>10 \%$ decrement in the percentage of predicted FVC represents a valid measure of disease progression ${ }^{[4]}$.

Clinical prediction models are statistical models that combine clinical findings from history, physical examination, and/or test results to estimate the probability of an outcome, usually a diagnosis or prognosis ${ }^{[2]}$ Baseline DLCO and CPI score also predicted survival ${ }^{[5]}$ ILD using the GAP model, a clinical prediction model based on sex, age, and lung physiology, that was previously validated in patients with idiopathic pulmonary fibrosis. ${ }^{[6]}$ The ILD-GAP model accurately predicts mortality in major chronic ILD subtypes and at all stages of disease ${ }^{[6]}$

The existing studies show that there are wide variations in the outcomes of the various types of ILD Studies assessing factors associated with mortality in ILDs are scarce in our setting . Data 
regarding the use of composite prediction models like Composite physiologic index and ILD Gap index are limited Prospective disease registries can provide better estimates of incidence and prevalence as well as insights to etiology, associated risks, natural history, and outcomes of a disease.

\section{Methodology}

Study design: Prospective Cohort study

Study setting: Dept of Pulmonary Medicine, Government Medical College Trivandrum.

Study period: Two years.

Study population: Patients with multidisciplinary diagnosis of ILD, attending Department of Pulmonary Medicine, Government Medical College Trivandrum,

Inclusion criteria: All patients diagnosed as ILD patients, who give consent to participate in the study.

Exclusion criteria: ILD Patients with Lung Cancer or Pulmonary Tuberculosis

\section{Objectives}

\section{Primary objective}

1.To compare the adverse outcome as defined by decline in FVC/ DLCO at 6 months in patients with Idiopathic pulmonary fibrosis(IPF) and other non IPF interstitial lung diseases presenting to Department of Pulmonary medicine, $\mathrm{MCH}$, Trivandrum for a period of two years.

Secondary objectives: 1 . To determine factors affecting adverse outcomes in interstitial lung diseases.2. To determine the prognostic value of composite physiologic index (CPI) and ILD GAP index in interstitial lung diseases.

Data collection A prospective cohort study over a period of two years conducted at Pulmonary medicine, Department, Medical College, Thiruvananthapuram. 164 consecutive patients with multidisciplinary diagnosis of interstitial lung disease were included in the study. Detailed history, spirometry, SPO2, DLCO, HRCT ,6 minute walk test, ECG,2D ECHO were done and recorded in duly filled proforma.ANA profile, serum angiotensin converting enzyme, serum calcium, 24 hour urinary calcium were estimated wherever it was indicated. Diagnosis of IPF was done after excluding the known causes and the HRCT findings suggestive of UIP pattern based on the current guidelines of the American Thoracic Society/European Respiratory Society CPI was calculated from spirometry and DLCO. ILD GAP index was also calculated. Patients were given appropriate therapy as per the clinical subtypes. Patients were followed up with clinical assessment and spirometry, and DLCO at 6 months. Outcome variables assessed were death and FVC decline $\geq 10 \% / \mathrm{DLCO}>15 \%$ at 6 months.

Institutional ethical committee clearance was obtained before data collection.

Statistical Analysis. Data were entered in Microsoft Excel and analyzed using Epi Info version 7. For descriptive statistics, quantitative variables were described by mean and standard deviation. Qualitative variables were described by percentage distribution. For inferential statistics between groups, comparison of qualitative variables were analysed by chi-square test and quantitative variables were compared by student $\mathrm{t}$ test. $\mathrm{P}$ value of less than 0.05 was considered as level of significance Survival pattern was assessed by Kaplan-meir survival plot.

\section{Results}

Fig 1. Proportion of IPF among Interstitial lung diseases

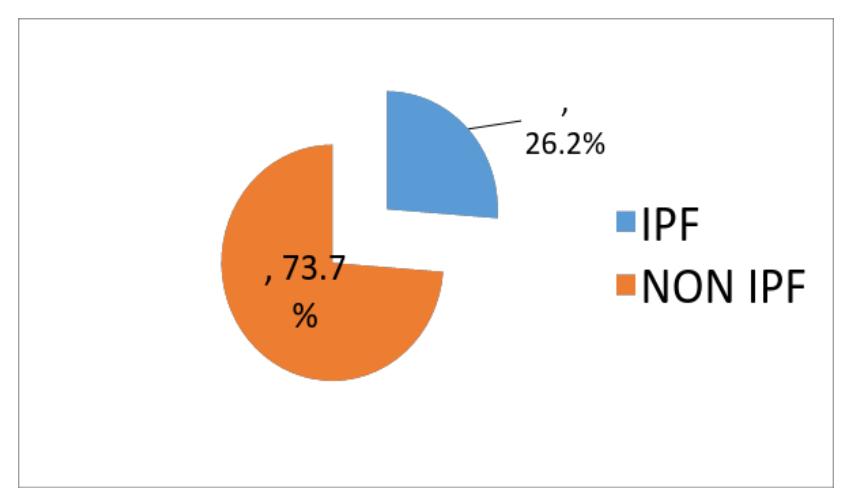




\section{JMSCR Vol||05||Issue||06||Page 23922-23929||June}

\section{Outcome Assessment}

1.Mortality at 6 months.

Out of the 164 patients studied, 25 patients (15.24\%), (95\% CI: $10.11 \%, 21.68 \%)$ died at 6 months of follow-up.

FIG 2: Survival analysis for different types of ILD (IPF vs Non IPF)

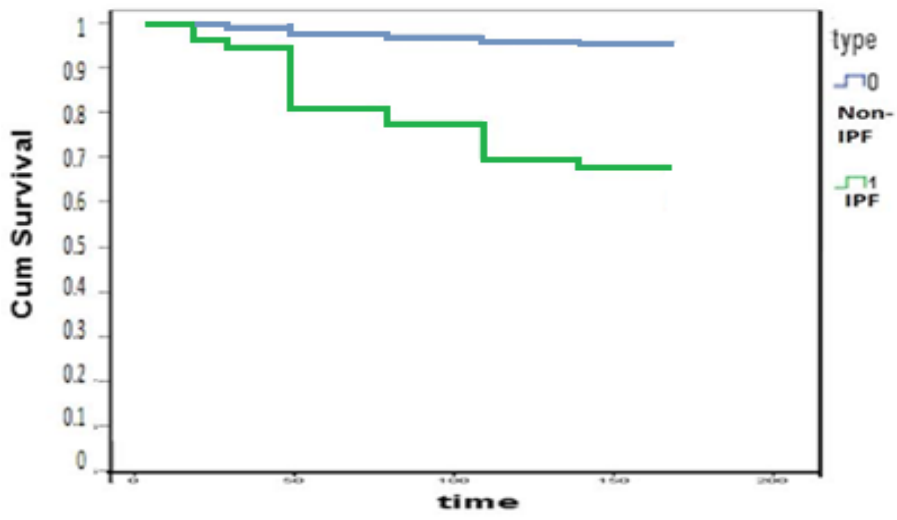

Mortality at 6 months was $41.86 \%$ in IPF group and, $5.79 \%$ Non-IPF group.

The difference in the proportion of mortality among IPF and other ILDs was statistically significant. (RR: $1.62,95 \%$ CI: $1.25,2.09$ ) with $\mathrm{p}$ value $<0.001$

2. Decline in $\mathrm{FVC} \geq 10 \%$ at 6 months.
Out of the 164 patients, 84 patients $(51.2 \%, 95 \%$ CI: $41.3 \%, 59 \%$ ) had a fall in FVC > $10 \%$ at 6 months.

Fig 3: proportion of FVC decline $\geq 10 \%$ among IPF and other ILDs.

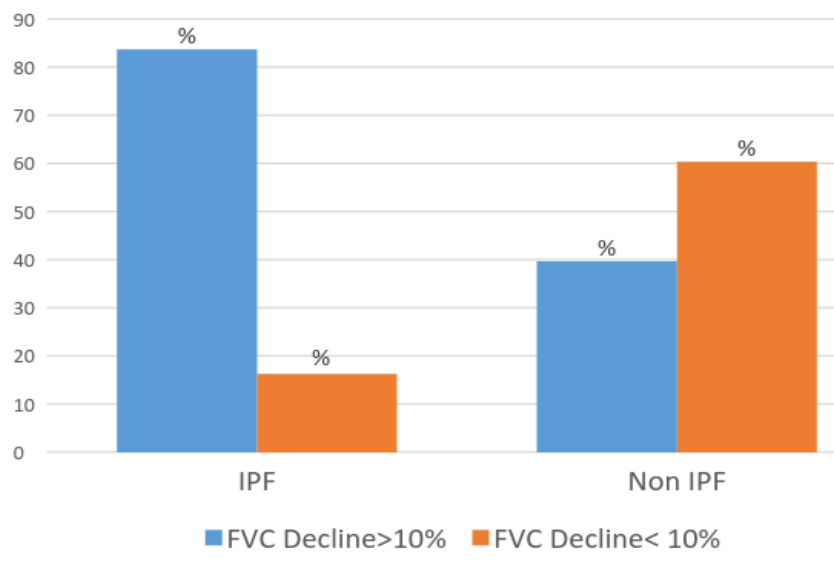

FVC decline was more in $\operatorname{IPF}(83.7 \%)$ compared to non-IPF(39.6\%). The difference in the proportion of FVC decline $\geq 10 \%$ in both groups was statistically significant (RR 1.59, 95\% CI: $1.3,1.94) \mathrm{p}$ value $<0.001$.

Factors Associated With Adverse Outcomes In Interstitial Lung Diseases.

1.Factors Associated With Mortality At 6 Months.

Table 1 : Factors associated with mortality in interstitial lung diseases(Bivariate analysis)

\begin{tabular}{|c|c|c|c|c|c|c|c|c|}
\hline p value & & $95 \% \mathrm{CI}$ & $\mathrm{RR}$ & & $\begin{array}{c}\text { Not } \\
\text { survived }\end{array}$ & Survived & \\
\hline 0.002 & & & & $\%$ & $\mathrm{n}$ & $\%$ & $\mathrm{n}$ & \\
\hline$<0.001$ & 1.75 & 1.15 & 1.4 & 68 & 17 & 23.02 & 32 & Age $>60$ \\
\hline$<0.001$ & 3.84 & 1.26 & 2.14 & 44 & 11 & 5.76 & 8 & Sex male \\
\hline$<0.001$ & 1.5 & 1.06 & 1.3 & 56 & 14 & 23.02 & 32 & BMI $<18.5$ \\
\hline$<$ & & & & & & & & Ever smoker \\
\hline$<0.001$ & 1.75 & 1.15 & 1.42 & 68 & 17 & 23.02 & 32 & Pulmonary \\
\hline 0.4 & 1.17 & 0.8 & 1.02 & 72 & 18 & 69.06 & 96 & Gypertension \\
\hline 0.003 & 1.4 & 1.1 & 1.26 & 88 & 22 & 48.92 & 68 & FVC $<50 \%$ \\
\hline$<0.001$ & 2.9 & 1.4 & 2.1 & 86.96 & 20 & 16.3 & 19 & DLCO $<40 \%$ \\
\hline$<0.001$ & 1.6 & 1.16 & 1.3 & 83.3 & 20 & 34.07 & 46 & 6 MWD $<250 \mathrm{~m}$ \\
\hline$<0.001$ & 1.75 & 1.18 & 1.4 & 76 & 19 & 25.9 & 36 & HRCT UIP pattern \\
\hline$<0.001$ & 1.9 & 1.3 & 1.6 & 100 & 24 & 34.9 & 40 & CPI score $>50$ \\
\hline$<0.001$ & 2.9 & 1.4 & 2.1 & 86.96 & 20 & 16.3 & 17 & ILD Gap index $>4$ \\
\hline
\end{tabular}

GERD symptoms were not significantly associated with mortality. On multivariate analysis using logistic regression, factors significantly associated with mortality were age > 60 years and type of ILD as idiopathic pulmonary fibrosis. 
2.Factors Associated with FVC Decline $>10 \%$ At 6 Months.

In bivariate analysis, factors associated with decline in FVC more than $10 \%$ at 6 months are age $>60, \mathrm{BMI}<18.5$, male sex, smoking history, presence of GERD symptoms, mean $\mathrm{SpO} 2<90 \%$, $\mathrm{DLCO}<40 \%$ predicted, $6 \mathrm{MWD}<250 \mathrm{~m}$, presence of pulmonary hypertension, UIP pattern in HRCT, CPI score > 50, ILD Gap index > 4.No statistically significant association with FVC decline $>10 \%$ at 6 months and $\%$ predicted FVC at the time of recruitment on bivariate analysis.

But on multivariate analysis using logistic regression, factors associated with more than $10 \%$ decline in FVC at 6 months are history of smoking and initial $\%$ predicted DLCO $<40 \%$.

Prognostic Value of Composite Prediction Models 1.Composite Physiologic Index (CPI)

The mean value of CPI was $50.18 \pm 11.6$. The mean value of CPI was higher among IPF patients $(56.5 \pm 8.9)$ compared to other ILDs $(47.41 \pm 11.7) . \mathrm{p}<0.004$.

Fig 4: predictive scores of mortality for composite physiologic index.

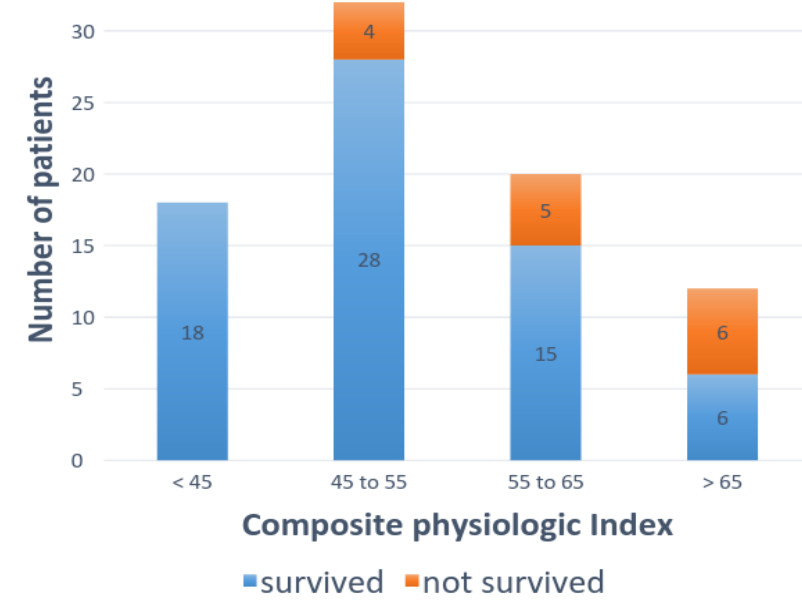

Higher CPI score $(60.92 \pm 5.6)$ was associated with poor survival at 6 months, compared to low CPI score $(47.9 \pm 11.4) \quad$ which was statistically significant. ( $\mathrm{p}$ value $<0.001$ )

\section{ILD GAP Index}

Out of the 164 ILD patients, 37 patients had ILD
Gap index > 4 (29.13\%), (95\% CI: $21.4 \%$, $37.8 \%$ ). $75.6 \%$ of IPF group had GAP Index $>4$ where as only $6.9 \%$ of non -IPF had $>4$ GAP Index.

The difference in proportion of patients with ILD Gap index $>4$ in both groups was statistically significant.( RR- 4.5, 95\% CI: 2.6, 11.4).p<0.005.

Fig 5: predictive score of mortality for ILD Gap index.

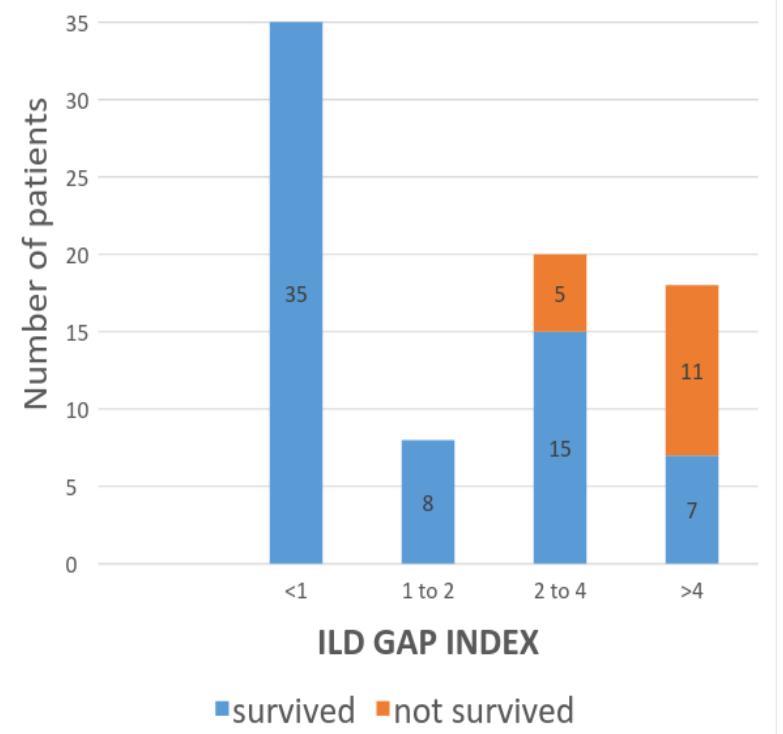

ILD Gap index > 4 is associated with increased mortality (RR 2.1, 95\% CI: 1.4, 2.9, p value < $0.001)$

Fig 6: ROC curve for ILD Gap index predicting mortality

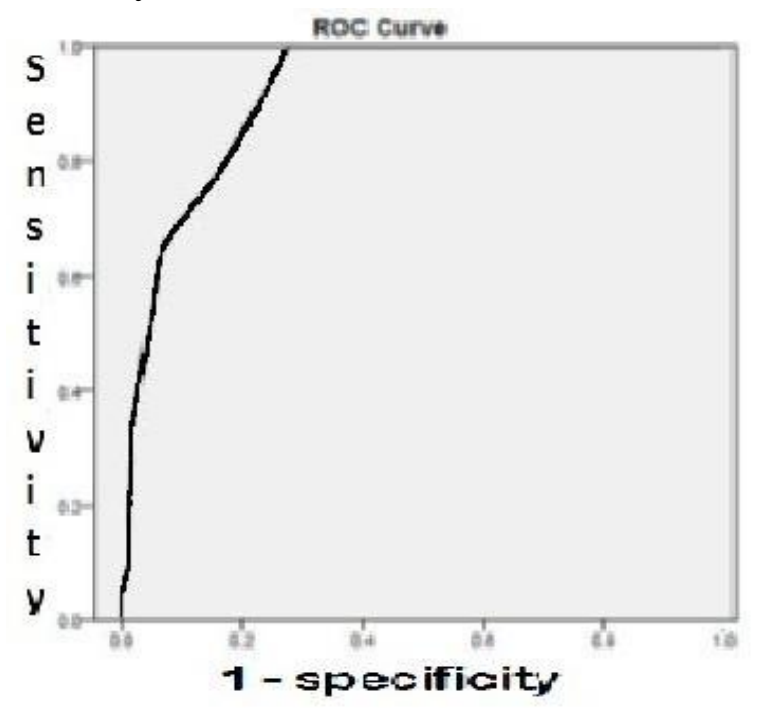

Area under curve: $\underline{0.912}$

(p. 0.0003). 
Fig 7; ROC Curve for CPI in predicting mortality.

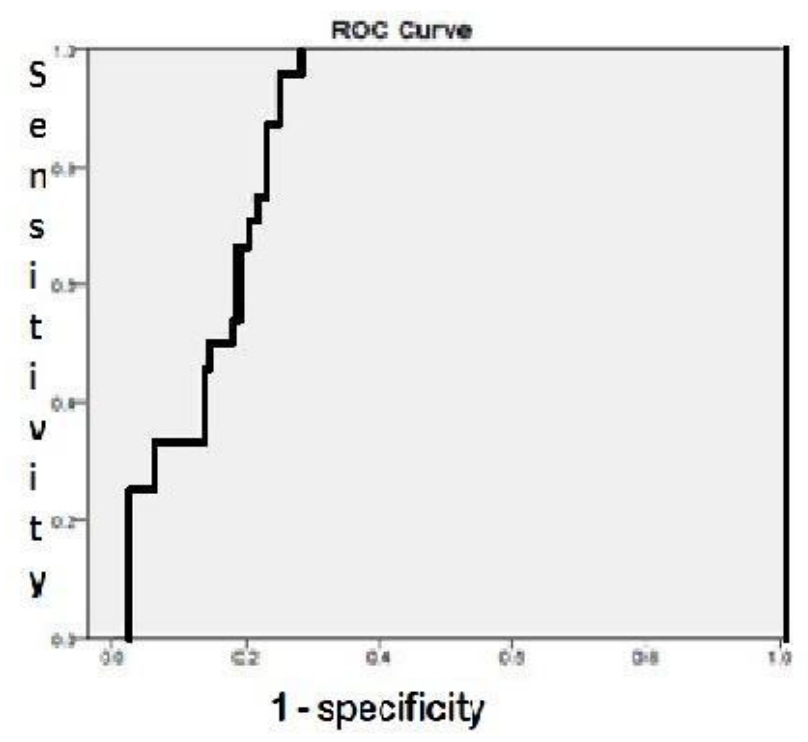

Area under curve: $\underline{0.856(\mathrm{p} 0.0002)}$

Comparing both ROC Curves, ILD Gap index is a better predictor of mortality than Composite physiologic index in interstitial lung diseases (AUROC 0.912 vs 0.856 for CPI).

\section{Discussion}

Risk prediction is challenging in chronic interstitial lung disease because of heterogeneity in disease ${ }^{[6]}$. To date we have limited information as to predictors of mortality in patients with IPF, and research in this area has failed to yield prediction models that can be reliably used in clinical practice to predict individual risk of mortality ${ }^{[2]}$ Usually FVC may be the most appropriate single prognostic parameter, given its ease of measurement, reproducibility, and ability to predict prognosis at baseline and over time, with even minor changes providing prognostic information $^{[2]}$ Clinical prediction models are statistical models that combine clinical findings from history, physical examination, and/or test results to estimate the probability of an outcome. ${ }^{[2]}$ The goal of this study is to find out clinical and physiological outcome OF ILD at 6 months follow up, factors that predict survival, and to evaluate prediction models like CPI and ILD- GAP Index in ILD.

We conducted a hospital based cohort study, in
164 patients diagnosed as ILD by multidisciplinary discussion.

Out of the 164 patients studied, 26.2\% were idiopathic pulmonary fibrosis (IPF) and $73.78 \%$ were non-IPF, similar to that reported by Sharma et al ${ }^{[16]}$. Somenath Kundu et al reported that IPF was found to be the most common ILD ( 38.04\%) followed by CTD -ILD( $31.5 \%){ }^{[17]}$.

In our study $15.2 \%$ of study population died at 6 months follow up, of which IPF had higher mortality than non-IPF. There was statistically significant difference in mortality among IPF and non-IPF ILDs $(41.8 \%$ vs $5.8 \%)$. Study by Amandine Vial et also highlighted the poor prognosis of IPF compared to other ILDs. ${ }^{[6]}$

In the study, factors associated with mortality in interstitial lung diseases are: age > 60, male gender, BMI < 18.5, smoking, presence of pulmonary hypertension, mean saturation $<90 \%$, $\%$ predicted $\mathrm{FVC}<50 \%$, \% predicted DLCO < $40 \%, 6$ Minute walk distance $<250 \mathrm{~m}$, at diagnosis, UIP pattern in HRCT, CPI score > 50 and ILDGAP index > 4On multivariate analysis, factors associated with mortality were age $>60$ years and IPF group. In a study by Assayag $D$ et al, significant predictors of mortality on multivariate analysis were older age, male gender, lower diffusion capacity for carbon monoxide, extent of fibrosis, and the presence of usual interstitial pneumonia pattern. ${ }^{[9]}$

In the study, $51.2 \%$ of patients had a fall in FVC $\geq$ $10 \%$ at 6 months. Factors that were associated with decline in FVC more than $10 \%$ at 6 months were similar to that associated with mortality in bivariate analysis, whereas on multivariate analysis ,history of smoking and initial \% predicted DLCO $<40 \%$ were significant.

Our study showed that FVC decline $>10 \%$ was more in IPF $(83.7 \%)$ compared to non-IPF (39.6\%). Dinesh Khanna et al reported that decline in $\mathrm{FVC} \%$ during the 12-month period was $12.8 \%$. $^{[18]}$

In our study, $\mathrm{FVC}<50 \%$ at baseline is associated with mortality similar to the finding reported by Solomon JJ that, a lower baseline \% predicted 
forced vital capacity and a $10 \%$ decline in FVC \% predicted from baseline to any time during follow up were independently associated with an increased risk of death ${ }^{[10]}$. King TE Jr $>10 \%$ decrement in the percentage of predicted FVC represents a valid measure of disease progression. ${ }^{[3]}$

In this study, composite prediction models like composite physiologic index and ILD Gap index were evaluated and the scores were higher among IPF patients, which again indicates a poor prognosis in IPF compared to other ILDs. Previous studies showed that the ILD-GAP model accurately predicts mortality in major chronic ILD subtypes and at all stages of disease ${ }^{[6]}$. Study by Charles Sharp et al also confirmed the validity of, GAP, and CPI as multidimensional indices in prognostication for IPF ${ }^{[7]}$. ILD Gap index is a better predictor of mortality than Composite physiologic index in interstitial lung diseases in our study, similar to the result of study by Hanna $\mathrm{M}$ et al. ${ }^{[5]}$

\section{Conclusion}

The factors associated with mortality in ILD were age $>60$ years, type of ILD as idiopathic pulmonary fibrosis and decline in $\mathrm{FVC} \geq 10 \%$ at 6 month.and FVC $<50 \%$ at baseline. The availability of reliable prediction models like composite physiologic index (CPI) and ILD Gap index can help in prognostication and making clinical decisions.

ILD Gap index was a better predictor of mortality than composite physiologic index.

\section{References}

1. William D. Travis, Ulrich Costabel, David M. Hansell , Talmadge E. King Jr.. An Official American Thoracic Society/European Respiratory Society Statement: Update of the International Multidisciplinary Classification of the Idiopathic Interstitial Pneumonias; American Journal of Respiratory and Critical Care MedicineVol. 188, No. 6 |
Sep

15 ,

2013

doi.org/10.1164/rccm.201308-1483ST

2. Brett Ley, Harold R. Collard, and Talmadge E. King Jr. Clinical Course and Prediction of Survival in Idiopathic Pulmonary Fibrosis American Journal of Respiratory and Critical Care MedicineVol. 183, No. 4 | Feb 15, 2011 https://doi.org/10.1164/rccm.2010060894CI

3. King TE Jr, Safrin S, Starko KM, Brown KK, Noble PW, Raghu G, Schwartz DA. Analyses of efficacy end points in a controlled trial of interferon-gamma1b for idiopathic pulmonary fibrosis.Chest2005 Jan;127(1):171-7.

DOI:10.1378/chest.127.1.171

4. Matthew R. Lammi, Robert P. Baughman, Surinder S. Birring, Anne-Marie Russell, Jay H. Ryu, Marybeth Scholand. Outcome Measures for Clinical Trials in Interstitial Lung Diseases; Curr Respir Med Rev. 2015; 11(2): 163-174. doi:10.2174/1573398X11666150619183527

5. Hanna M. NurmiEmail author, Minna K. Purokivi, Miia S. Kärkkäinen, Are risk predicting models useful for estimating survival of patients with rheumatoid arthritis-associated interstitial lung disease? BMC Pulmonary Medicine BMC series - open, inclusive and trusted201717:16DOI: $\quad 10.1186 / \mathrm{s} 12890$ 016-0358-2

6. Ryerson CJ, Vittinghoff E, Ley B, Lee JS, Mooney JJ, Jones KD, Elicker BM, Wolters PJ, Koth LL, King TE Jr, Collard HR Predicting survival across chronic interstitial lung disease: the ILD-GAP model. CHEST2014 Apr;145(4):723-728. doi: 10.1378/chest.13-1474.

7. Huzaifa I. Adamali, and Ann B. Millarø A comparison of published multidimensional indices to predict outcome in idiopathic pulmonary fibrosis ERJ Open Res. 2017 Jan; 3(1): 00096-2016. . doi: 
10.1183/23120541.00096-2016

8. Amandine Vial-Dupuy, Olivier Sanchez, Liath Guetta, Benoit Douvry, Karine Juvin, Delphine Wermert, Emmanuel Guerot, Dominique Israel-Biet.Outcome of patients with interstitial lung disease admitted into ICUEuropean Respiratory Journal 2011 38: 4862; DOI:

9. Assayag D, Lubin M, Lee JS, King TE, Collard HR, Ryerson CJ. Predictors of mortality in rheumatoid arthritis-related interstitial lung disease. Asian Pacific Society of Respirology 2014 May;19(4): 493-500. doi: 10.1111/resp.12234.

10. Solomon JJ, Chung JH, Cosgrove GP, Demoruelle MK, Fernandez-Perez ER, Fischer A Frankel SK, Hobbs SB Predictors of mortality in rheumatoid arthritis-associated interstitial lung disease. .ERJ 2016 Feb;47(2):588-96. doi: 10.1183/13993003.00357-2015. Epub 2015 Nov 19.

11. Su-Ying Low Using the ILD-GAP model to predict mortality in chronic interstitial lung disease European Respiratory Journal 2015 46: PA3824; DOI: 10.1183/13993003.

12. Sang Hoon Lee, Song Yee Kim, Dong Soon Kim. Predicting survival of patients with idiopathic pulmonary fibrosis using GAP score: a nationwide cohort study; Respiratory Research201617:131DOI: 10.1186/s12931-016-0454-0

13. Julie Morisset, Eric Vittinghoff , Bo Young Lee, Roberto Tonelli, Xiaowen $\mathrm{Hu}$ Predicting Mortality in Patients with Rheumatoid Arthritis Related Interstitial Lung Disease: Expanding The GAP Model ,American Journal of Respiratory and Critical Care Medicine 2016;193:A4288

14. Joshua Mooney , Karina Raimundo , Eunice Chang, Michael S. Broder, Mortality, Costs, and Length of Stay in Patients with Idiopathic Pulmonary
Fibrosis American Journal of Respiratory and Critical Care Medicine 2016;193:A4286

15. Christopher J Ryerson, Eric Vittinghoff Christopher J Ryerson. Predicting Survival Across Chronic Interstitial Lung Disease The ILD-GAP Model;Chest 145(4) · Oct. 2013 DOI: 10.1378/chest.13-1474 .

16. Laying the ground for research of interstitial lung disease in our country: ILD India registry Lung India 31(4):320-2 - October 2014 DOI: 10.4103/0970-2113.142091.

17. Somenath Kundu, Subhra Mitra, Joydeep Ganguly, Subhasis Mukherjee, Souvik Ray Ritabrata Mitra. Spectrum of diffuse parenchymal lung diseases with special reference to idiopathic pulmonary fibrosis and connective tissue disease: An eastern India experience Lung India 2014;31:35460.

18. Dinesh Khanna, ${ }^{1}$ Chi-Hong Tseng, ${ }^{2}$ Niloofar Farmani, ${ }^{3}$ Virginia Steen Clinical Course of Lung Physiology in Patients with Scleroderma and Interstitial Lung Disease: Analysis of the Scleroderma Lung Study Placebo Group; Arthritis Rheum. 2011 Oct; 63(10): 3078-3085. doi: 10.1002/art.30467. 